\title{
Quantification of Diabetic Maculopathy by Digital Imaging of the Fundus
}

\author{
R. P. PHILLIPS ${ }^{1}$, T. SPENCER ${ }^{2}$, P. G. B. ROSS ${ }^{2}$, P. F. SHARP ${ }^{2}$, J . V. FORRESTER ${ }^{1}$ \\ Aberdeen
}

\begin{abstract}
Summary
Measurement of the extent of diabetic retinopathy is an essential part of assessing the efficacy of local or systemic treatment regimens. Current clinical studies use empirical grading of retinopathy which is performed by a trained observer using standard photographs. This method is relatively arbitrary, as well as time consuming and vulnerable to observer error.

We have developed a digital fundus imaging system and image processing programs which provide objective, quantitative measures of macular oedema, retinal exudates, and microaneurysms in diabetic retinopathy. Using fluorescein angiograms, the degree of macular oedema is quantified both in terms of area of fundus involved and severity of oedema by analysis of the temporal changes in intensity of fluorescence. Fluorescein angiograms are also used for the detection and counting of microaneurysms, by a combination of shade correction, matched filtering, and shape algorithms. For detection and measurement of retinal exudates, a colour transparency projected through a red free filter is analysed using a combination of shade correction and thresholding techniques.

The system described is in clinical use, and has potential for a wide variety of applications. With further development, digital analysis of fundus images should supercede the currently used manual semi-quantitative methods, providing faster, more accurate, objective quantitative results.
\end{abstract}

The purpose of quantifying the visible retinal pathological features of diabetic retinopathy is to be able to follow the progress of the retinal disease either with or without treatment. In general terms, quantification of lesions consists either of object counting or area measurement.

Manual counts of small numbers of well defined objects can be accurate and reproducible, but reliability declines as the objects become less sharply defined and larger in number. Area measurements are less well performed manually. Many of the lesions are of irregular shape, and may have ill defined margins, making inter and intra observer variations more likely.

The classification systems currently in use recognise these problems, ${ }^{1,2}$ and make no attempt to measure precisely areas of leakage or hard exudates. Instead, area measurements are related to the optic disc diameter or to a percentage of a given field of photo-

From: ${ }^{1}$ Department of Ophthalmology, ${ }^{2}$ Department of Bio-Medical Physics, Medical School, University of Aberdeen, Foresterhill, Aberdeen AB9 2ZD

Correspondence to: Dr. Phillips, Department of Ophthalmology, Medical School, University of Aberdeen, Foresterhill, Aberdeen AB9 2ZD. 
graphy. ${ }^{2}$ Grading of severity of retinopathy is currently performed by comparison of predefined photographic fields with a standard set of photographs. ${ }^{1}$ The entire photographic field comprises the 'region of interest', and thus varies in pohotographs taken on different occasions. Photographic variations in focus, illumination, magnification, and angle of photography may all affect the contrast of a lesion, and thus affect its 'detectability'.

The objectivity of computerised quantitative image analysis lends itself to the study of diabetic maculopathy, and in particular to the quantification of microaneurysms, exudates, and macular leakage. Microaneurysm counts are a recognised predictor for the progression of retinopathy. ${ }^{3,4}$ Manual techniques for counting microaneurysms depend on the use of a grid by a trained observer, ${ }^{4,5}$ and may take up to forty minutes to perform. ${ }^{5,6}$ Badouin et al have described an automated computerised method for detecting and quantifying microaneurysms using mathematical morphological analysis of fluorescein angiograms. ${ }^{6}$

Computerised methods of measuring exudates have been described. ${ }^{7,8,9}$ At best these are semi-automated, requiring user interaction at thresholding stages, thus reducing the objectivity of the techniques.

In current studies of diabetic retinopathy the methods used for quantifying macular oedema are semi-quantitative ${ }^{1,2}$ and are dependent on evaluation by a trained observer. No attempt at a precise measurement of the area of macular thickening or fluorescein leakage is made. Tamura's computerised method for measuring the area of fluorescein leakage ${ }^{10}$ has not found clinical application.

We describe in outline a digital imaging system and suite of programs designed to detect and quantify microaneurysms, exudates, and hyperfluorescent macular leakage in diabetic retinopathy. In each case the analysis is automated apart from the choice by the operator of the region to be analysed. We outline the criteria and procedures necessary for obtaining reproducible results. The results of our initial studies of the accuracy and reproducibility of these techniques are summarised and compared with other methods of quantifying the features of diabetic retinopathy.

\section{Methods}

Colour slides or fluorescein angiogram nega- tives were projected onto a matt white screen and viewed by a Charge Coupled Device (CCD) video camera. Room lighting, camera aperture, distance between CCD camera and screen, and camera angle were standardised. The image was digitised by and stored in a frame grabber installed in an IBM PC-AT compatible microcomputer with 20 MByte hard disk. Image processing was performed by the PC, the frame grabber, and by auxiliary frame processor and transputer boards installed in the PC. Control of image acquisition and processing was by custom designed software written in $\mathrm{C}$, incorporating a commercially available image processing library. A more detailed description of the system is given elsewhere. ${ }^{11,12}$

\section{Macular leakage}

The rationale behind this method is that at any location in a normal angiogram, the level of fluorescence would always be expected to reach a peak during initial dye transit and then decrease with time. Where leakage is present the rate at which fluorescence decreases may be reduced, or there may even be an increase in fluorescence. On this basis the technique differentiates leakage from normal structures with above average fluorescence e.g. vessels. Our technique for estimating hyperflourescent leakage has been reported ${ }^{11}$ and is described briefly below.

Two frames from a fluorescein angiogram were chosen for the analysis. The first corresponded to completion of venous filling, usually between 15 and 30 seconds, at which time no significant leakage had occurred. The second, usually between 250 and 300 seconds, gave sufficient time for leakage to have taken place. The images were aligned manually during acquisition, and particular attention was paid to ensure accurate projection of the images onto the screen. Fine correction of linear misalignment was then carried out with a software routine using markers added manually to the digitised images.

The region of interest was a square centred on the fovea. By comparing matching spatial locations in the two frames, a gradient of fluorescence intensity was calculated. By experimentation on a number of normal angiograms, a threshold gradient value was 
chosen. Pixels whose gradient was less than this value were identified as corresponding to leakage.

For display purposes each pixel demonstrating leakage was assigned a colour corresponding to the severity of leakage and the area of leakage was overlaid on the original late frame (Fig. 3c). The area of leakage, in terms of the number of affected pixels, and the sum of their corresponding 'leakage values' were then calculated The analysis took approximately five minutes. The accuracy and reproducibility of the system were tested.

\section{Exudates}

As retinal exudates appear 'whiter', i.e. have a higher gray level value than the surrounding normal retina, a gray level thresholding technique $^{13}$ was used to segment and quantify the exudates. Our method has been reported in detail elsewhere,$^{12}$ and is summarised below.

Before thresholding was performed the digitised colour slide was processed to eliminate photographic non-uniformities in illumination ('shade correction'), and the contrast of the exudates was then enhanced. ${ }^{14}$ The exudates were detected by thresholding, the threshold being calculated by the computer on the basis of the distribution of gray levels in the image. Thus the only user interaction required during the runining of the program is choice of the region of interest. The analysis takes approximately three minutes. Reproducibility was assessed using a number of different colour slides and regions of interest.

\section{Microaneurysms}

Microaneurysms appear as small rounded hyperfluorescent objects in the arteriovenous, and early venous phases of fluorescein angiograms. They are of similar gray level to surrounding highly fluorescent structures such as vessels. Detection of mircoaneurysms can not therefore depend solely on gray level thresholding techniques. We have used a combination of shade correction, matched filtering, ${ }^{15}$ and shape operators ${ }^{16}$ to detect and count microaneurysms in a region of interest within the macular area. The analysis takes between five and ten minutes. Fourteen regions of interest in eight different angiograms have each been analysed on five differ- ent, non-consecutive, occasions in order to assess reproducibility. Accuracy in terms of sensitivity and specificity was assessed by one of the authors (RPP) hand counting the microaneurysms for each region analysed, and comparing the manual counts and positions with those of the computer. A more detailed masked study comparing manual counts by a number of observers with automated computer counts is in progress.

\section{Results}

\section{Macular leakage}

The accuracy of the technique was estimated to be approximately $97 \%$. The coefficient of variation for reproducibility varied between $27 \%$ for small patchy areas of leakage, to less than $6 \%$ for large confluent areas of leakage.

Figure 1 illustrates early and late angiographic frames, and the resultant image. The late frame shown is not of ideal quality, being of poor contrast, with loss of the anatomical detail required for good image registration. This image has been chosen in order to illustrate the features of an image which make computerised quantitative analysis less reliable. For the reasons outlined above the area of leakage is slightly underestimated (see Discussion).

\section{Exudates}

The coefficient of variation for reproducibility varied between $17 \%$ for small scattered exudates to less than $3 \%$ for larger more confluent areas of exudates. A typical colour slide and result are shown in Figure 2.

\section{Microaneurysms}

Using a single observer as the 'gold standard', sensitivity of our computerised technique was between $82 \%$ and $93 \%$, and specificity $93 \%$ or better. The coefficient of variation for reproducibility was between $7 \%$ and $14.5 \%$ for microaneurysm counts. Seventy-five per cent or more of the microaneurysms were detected in the same position on repeated analyses of the same region of the same angiographic frame (reproducibility of position). An example of a typical result is shown in Figure 3.

\section{Discussion}

The cognitive abilitity of the human observer 

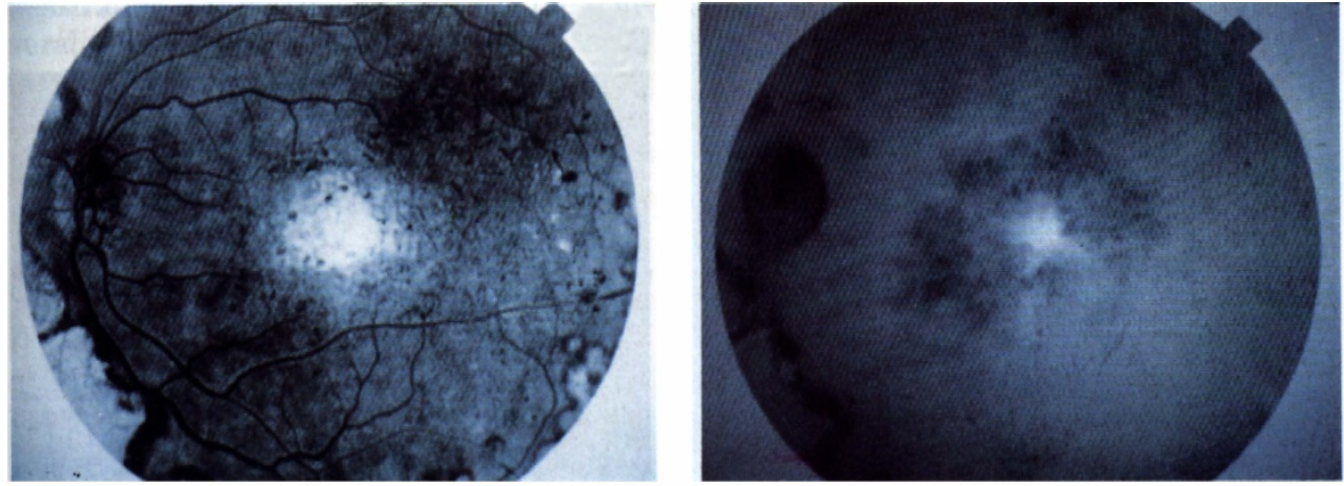

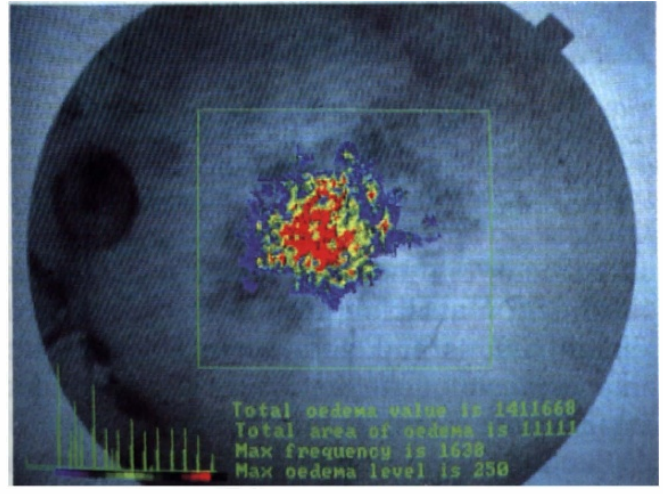

is capable of compensating for some of the photographic variations in fundus images, but may not do so reproducibly. A computer has no cognitive function, obeying only the instructions given to it (the program), but will always produce an identical result in analysing

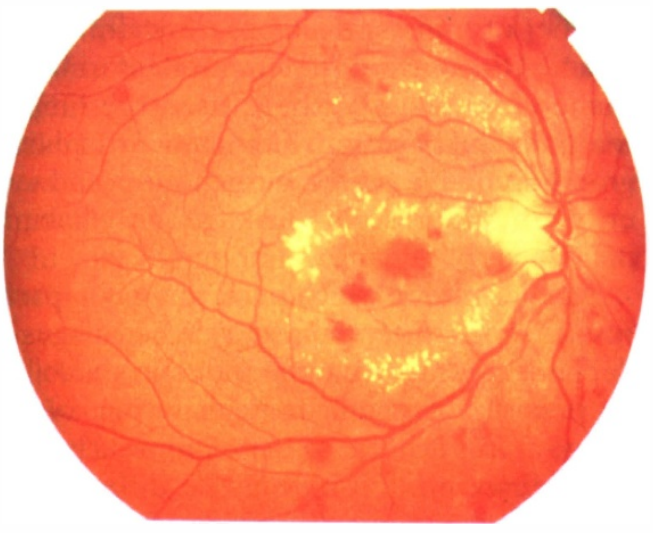

Fig. 1. (a) Early frame of angiogram. (b) Late frame of angiogram. Note that the contrast of this image is poor and most of the anatomical detail is absent. This frame has been used in order to demonstrate the effect of poor quality images on quantitative imaging (see Discussion). (c) Result of quantification of macular leakage with the region of interest outlined by the green box. Due to the poor quality of the late frame the area of leakage is slightly underestimated (see Discussion).

an identical large set of complex data i.e. an ophthalmic image. For counting large or small numbers of objects, and for area measurements, a computer is therefore capable of $100 \%$ reproducibility under ideal circumstances. In practical terms it is the quality of

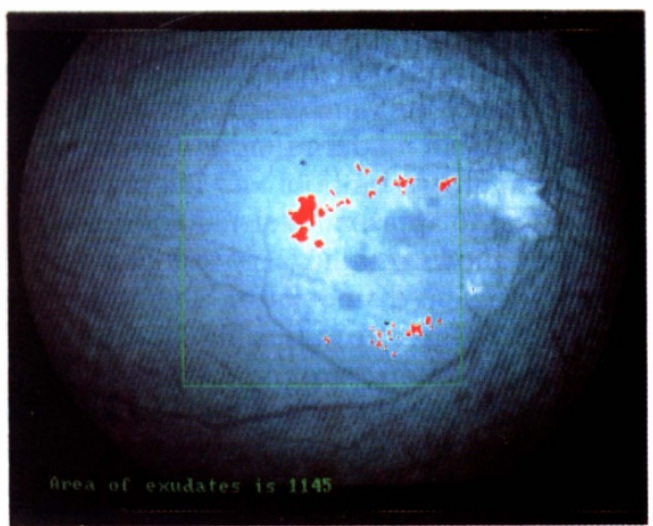

Fig. 2. (a) Colour fundus photograph showing diabetic exudates. (b) Result of detection and quantification of exudates with the region of interest outlined by the green box. 

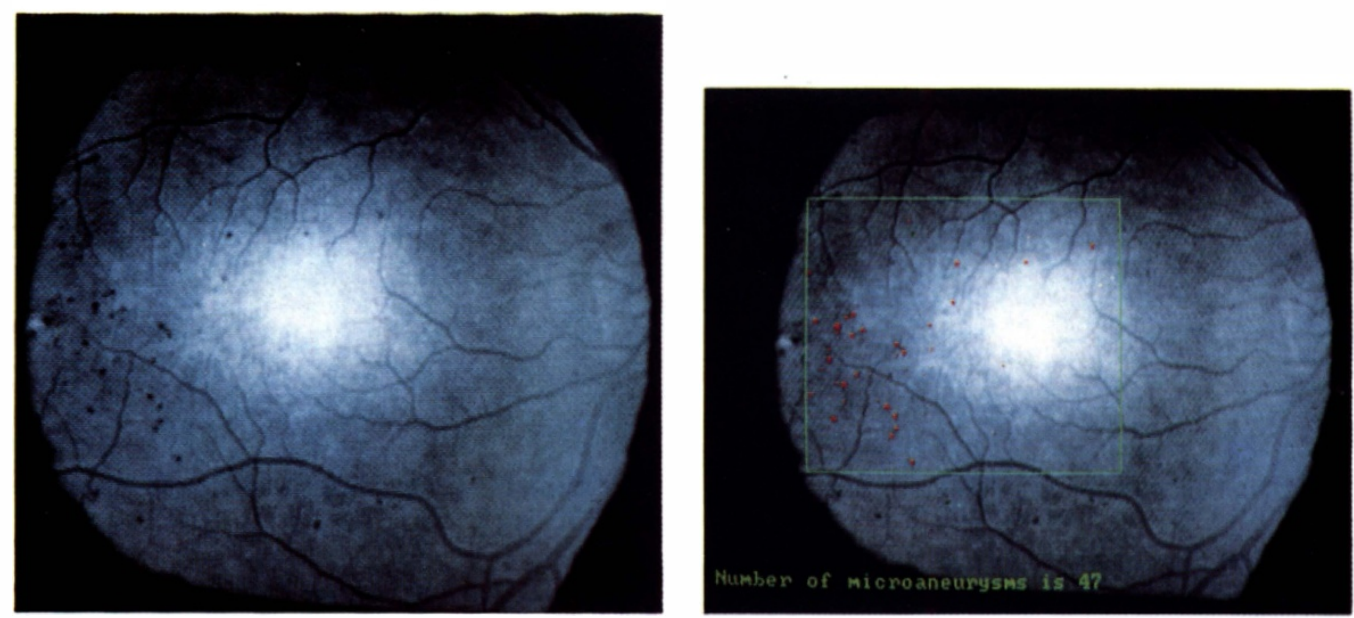

Fig. 3. (a) Early frame of fluorescein angiogram showing numerous microaneurysms. (b) Result of microaneurysm analysis showing the detected microaneurysms mapped in position and the count displayed. Note that there is a false negative adjacent to the large vessel in the lower temporal part of the region of interest (outlined by the green box) (see Discussion).

the input data which determines the reproducibility of computerised quantification of diabetic retinopathy.

When comparing manual with computerised methods of quantifying retinal pathology it is important to realise the differences which exist in the images which are presented to the human observer and the computer respectively. A photograph and a computerised image both consist of a finite number of 'dots'. In a photograph these dots correspond to the grain of the photographic emulsion, whereas a digital image is composed of 'picture elements' or pixels. The number of 'dots' for a given area decides the resolution of an image. Most digitising systems in current use produce an image of $512 \times 512$ pixels resolution. The black and white film used for fluorescein angiography in modern fundus cameras gives a maximum resolution of 164 lines $/ \mathrm{mm}$, equivalent to approximately $2000 \times 2000$ pixels. This allows resolution of lesions approximately $5 \mu \mathrm{m}$ in diameter for a photographic field of 30 degrees. Colour film gives a maximum resolution of approximately 90 lines $/ \mathrm{mm}$, equivalent to about $1100 \times 1100$ pixels.${ }^{17} \mathrm{~A}$ digitised fluorescein angiogram frame therefore contains only $\frac{1}{16}$ of the information contained in the original frame, and is capable of resolving lesions of approximately $20 \mu \mathrm{m}$ in diameter. Despite the lower resol- ution comparisons of qualitative diagnostic information available from directly acquired digital fundus and fluorescein angiographic images, and conventional photographic images have been favourable. ${ }^{18,19}$

Fundus fluorescein angiography is a dynamic investigation. Video fluoroscopy demonstrates this feature and has been used in qualitative analysis of angiograms. ${ }^{20} \mathrm{~A}$ fluorescein angiogram contains information of three types: spatial, intensity and temporal. Routinely, fluorescein angiograms are presented as a montage of a number of frames at fixed time intervals during the investigation. Photographic artefact usually reduces the number of frames which are clinically useful. Much of the dynamic or temporal information is therefore lost. We have used the limited temporal information in a standard photographic sequence to measure the area and severity of fluorescein leakage in diabetic maculopathy.

Any technique which performs a comparative analysis of temporally related images is dependent for its accuracy and reproducibility on precise alignment or 'registration' of the images. This is because the temporal changes in fluorescein intensity may be different for narrowly separated spatial locations of the fundus, depending mainly on vocal vascular pattern and pigmentation. ${ }^{21}$ Only with good 
registration is it possible to separate the temporal effects on fluorescein intensity from the spatial ones. Misregistration may be linear, rotational, geometric, or magnificationrelated, or a combination of all four. It may occur at the time of photography, and at the time of acquisition and digitisation (where slides are being used). Registration also requires features of the image as reference points. In late frames of the angiogram, particularly where the inner blood retina barrier is severely damaged, fluorescein leakage into the vitreous may severely reduce the quality and contrast of the angiographic frame (Fig. 3c). The reference points required for registration are lost and the intensity information is altered.

Our present method for measuring leakage attempts to minimise misregistration at the digitisation stage by a carefully standardised set-up routine. Any geometric misregistration contained within the photographs cannot be corrected by our simple manual alignment technique. The topic of computerised image registration is a large and complex one, and is beyond the scope of this article, but we are investigating a number of methods which could be incorporated into our present programs. It can be seen from the above discussion that our method for measuring leakage is therefore the best approximation to the ideal that is currently available with our prototype system. We feel that it improves on previously reported computerised methods ${ }^{10}$ and offers greater precision in measuring area and severity of leakage than manual methods. With improvements in hardware it will be possible to analyse a large number of directly acquired digital angiographic frames and make fuller use of the dynamic features of fluorescein angiography to provide quantitative information.

Ideally temporal changes in intensity during angiography should relate only to changes in fluorescein concentration and not to changes in illumination of the fundus. Lighting irregularities may markedly alter the contrast of an image or some of its features. Standardisation of room lighting conditions at the time of angiography is important and is easily achieved. Standardisation of fundus illumination is less straightforward. Maintain- ing a constant camera angle during angiography will eliminate most of the inconsistency produced by intraocular light reflections. The reflectance characteristics of every fundus differ according to the degree of pigmentation ${ }^{21}$ and this itself may alter the intensity information received by the camera. A measure of fundus reflectance at the appropriate wavelengths can be made using the digital imaging system and should be included in the standardisation of lighting conditions for angiography and photography. Where projected slides are being digitised room lighting must be standardised at the time of projection, and vignetting produced by the projection illumination system must be accounted for. The set-up procedure for our system attempts to minimise these effects.

Provided good quality images are chosen for input, and digitised under carefully standardised conditions, acceptable results for the detection and quantification of microaneurysms, exudates and macular leakage are produced. For our microaneurysm analysis the sensitivity of $82 \%$ and specificity of $93 \%$ improve on those reported by Badouin et al. ${ }^{6}$ of $70 \%$ and $85 \%$ respectively for his automated computerised method. Reproducibility of count was better than $85 \%$ which compares favourably with the $87.4 \%$ for intra-observer reproducibility, and $62.3 \%$ inter-observer reproducibility reported by manual counting. ${ }^{5,6}$ Reproducibility of position of better than $75 \%$ is an improvement over the $42-71 \%$ reported by Badouin $e t$ $a l .{ }^{5}$ for manual counting. Our exudate analysis has the advantage over previously reported methods of being automated in its choice of thresholds. We consider reproducibility with coefficient of variation in the range $3-17 \%$ to be acceptable. Our method of using temporal information to measure macular leakage from fluorescein angiograms is novel.

Assessment of the accuracy of a computerised method for quantitative fundus analysis is difficult because it requires knowledge of the 'rightness or wrongness' of the answer produced by the computer. A 'gold standard' is required where the 'correct' answer is determined by an independent method of known high accuracy and reproducibility. For quantification of diabetic retinopathy such methods 
do not exist. During the development of computer qanalysis methods the final arbiter is the researcher or the clinician deciding whether a result 'seems reasonable'. We have attempted to verify the accuracy of our methods. ${ }^{11}$ For digital imaging of the retina to be useful as a means of quantifying retinal pathology it must confer advantages over manual methods in terms of speed, precision, accuracy and reproducibility. The algorithms used should be sufficiently robust to meet these criteria on a wide variety of images.

In summary, the technology is now available to look at all types of ophthalmic images in a new way, be they colour or black and white. By analysing the intensity and temporal information contained within the images it is now possible to extract quantitative data from them. The way forward in quantitative fundus image analysis lies in providing good quality images to the computer under carefully standardised conditions. The potential for digital imaging of the fundus as a clinical tool is enormous. High quality hardcopy of digital images can be produced within minutes, along with the appropriate quantitative analysis. This eliminates the delays incurred by film development. Images and angiographic sequences can be archived in data retrieval systems for instant recall, analysis, and inclusion in data and knowledge bases.

\section{Conclusion}

We have described a low cost prototype digital imaging system on which we have investigated the quantitative information contained within fundus images. We have applied our results in the development of programs to detect and quantify microaneurysms, exudates and macular leakage in diabetic retinopathy. The applications described in this paper represent a small selection of those available by digital imaging of the fundus. We believe that with appropriate development quantitative analysis of fundus images will soon become a powerful clinical tool.

This research was funded by grants from the T.F.C. Frost Charity, the Scottish Home and Health Department, and the Grampian Health Board. We are grateful for the support of Professor J. Mallard, and Dr. P. Undrill of the Department of Bio-Medical Physics.
Key Words: Diabetic retinopathy; Computerised image analysis; Macular oedema.

\section{References}

${ }^{1}$ Diabetic Retinopathy Study: Report 7: A modification of the Airlie House classification of diabetic retinopathy. Invest Ophthalmol Vis Sci 1981, 21: $210-26$

${ }^{2}$ Smith RT, Lee CM, Charles HC, Farber M, CunhaVaz JG: Quantification of diabetic macular oedema. Arch Ophthalmol 1987, 105: 218-22.

${ }^{3}$ Kohner EM and Sleightholm M: The KROC collaborative study group. Does microaneurysm count reflect severity of early diabetic retinopathy? Ophthalmology 1986, 93: 586-9.

${ }^{4}$ Klein R, Meuer SM, Moss SE, Klein BE: The relationship of retinal microaneurysm counts to the 4-year progression of diabetic retinopathy. Arch Ophthalmol 1989, 107: 1781-5.

${ }^{5}$ Badouin C, Maneschi F, Quentel G, Soubrane G, Hayes T, Jones G, Coscas G, Kohner EM: Quantitative evaluation of fluorescein angiograms: Microaneurysm counts. Diabetes 1983, 32: 8-13.

${ }^{6}$ Baudoin CE, Lay BJ, Klein JC: Automatic detection of microaneurysms in diabetic fluorescein angiography. Rev Epidem et Sante Publ 1984, 32: 254-61.

${ }^{7}$ Ward NP, Tomlinson S, Taylor CJ: Image analysis of fundus photographs. The detection and measurement of exudates associated with diabetic retinopathy. Ophthalmology 1989, 96: 80-6.

${ }^{8}$ Sleightholm MA, Arnold JV, Aldington SJ, Kohner EM: Computer aided digitisation of fundus photographs. Clin Phys Physiol Meas 1984, 5: 295-301.

${ }^{9}$ Gilchrist J: Analysis of early diabetic retinopathy by computer processing of fundus images - a preliminary study. Ophthal Physiol Opt 1987, 7: 393-9.

${ }^{10}$ Tamura S, Tanaka K, Hashi M et al: Analysis of fluorescence fundus angiographies. National Convention of I.P.S.J. 1977, 673-4.

${ }^{11}$ Phillips RP, Ross PGB, Tyszka M, Sharp PF, Forrester JV: Detection and quantification of hyperfluorescent leakage by computer Analysis of fundus fluorescein angiograms. Graefe's Arch Glin Exp Ophthalmol (Submitted).

${ }^{12}$ Phillips RP, Ross PGB, Sharp PF, Forrester JV: Automated detection and quantification of retinal exudates. Am J Ophthalmol (Submitted).

${ }^{13}$ Rosenfeld A and Kak AC: Chapter 10; Digital Picture Processing. Orlando, Academic Press. 1982, pp. 61-73.

${ }^{14}$ Rosenfeld A and Kak AC: Chapter 6; Digital Picture Processing. Orlando, Academic Press. 1982, pp. 237-250.

${ }^{15}$ Rosenfeld A and Kak AC: Chapter 9; Digital Picture Processing. Orlando, Academic Press. 1982, pp. 42-46.

${ }^{16}$ Rosenfeld A and Kak AC: Chapter 11; Digital Picture Processing. Orlando, Academic Press. 1982, pp. 191-275. 
${ }^{17}$ Nielsen NV: The normal fundus fluorescein angiogram and the normal fundus photograph. Acta Ophthalmologica 1986, (suppl 180).

${ }^{18}$ Friberg TR, Rehkopf PG, Warnicki JW, Eller AW: Use of directly acquired digital fundus and fluorescein angiographic images in the diagnosis of retinal disease. Retina 1987, 7: 246-51.

${ }^{19}$ Seeley GW, Craine ER, Fryczkowski MD: Compari- son of conventional fluorescein angiography film images with a cathode ray tube display. Arch Ophthalmol 1989, 107: 227-31.

${ }^{20}$ Haining WM: Video fundoscopy and fluoroscopy. $\mathrm{Br}$ J Ophthalmol 1981, 65: 702-6.

${ }^{21}$ Delori FC and Pflibsen KP: Spectral reflectance of the human ocular fundus. Applied Optics 1989, 28: 1061-77. 